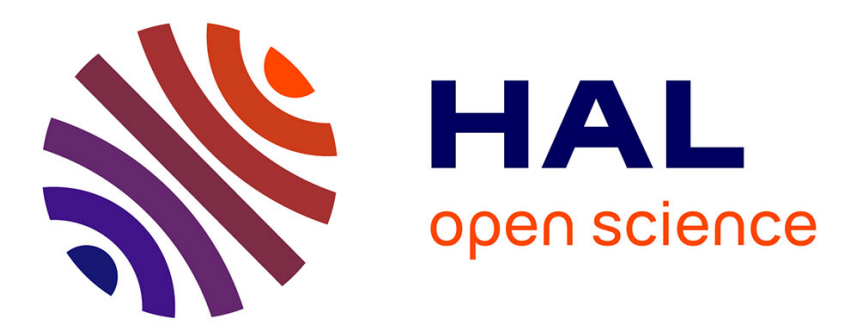

\title{
3D Image Analysis and Artificial Intelligence for Bone Disease Classification
}

Abdurrahim Akgundogdu, Rachid Jennane, Gabriel Aufort, Claude-Laurent Benhamou, Osman Nuri Ucan

\section{To cite this version:}

Abdurrahim Akgundogdu, Rachid Jennane, Gabriel Aufort, Claude-Laurent Benhamou, Osman Nuri Ucan. 3D Image Analysis and Artificial Intelligence for Bone Disease Classification. Journal of Medical Systems, 2010, 34 (5), pp.815-828. 10.1007/s10916-009-9296-3 . hal-00655386

\section{HAL Id: hal-00655386 https://hal.science/hal-00655386}

Submitted on 1 Jan 2012

HAL is a multi-disciplinary open access archive for the deposit and dissemination of scientific research documents, whether they are published or not. The documents may come from teaching and research institutions in France or abroad, or from public or private research centers.
L'archive ouverte pluridisciplinaire HAL, est destinée au dépôt et à la diffusion de documents scientifiques de niveau recherche, publiés ou non, émanant des établissements d'enseignement et de recherche français ou étrangers, des laboratoires publics ou privés. 


\title{
3D IMAGE ANALYSIS AND ARTIFICIAL INTELLIGENCE FOR BONE DISEASE CLASSIFICATION
}

\author{
Abdurrahim Akgundogdu ${ }^{1}$, Rachid Jennane ${ }^{2}$, Gabriel Aufort ${ }^{2}$, Claude Laurent Benhamou ${ }^{3}$, \\ Osman Nuri Ucan ${ }^{1}$ \\ ${ }^{1}$ Istanbul University, Department of Electrical and Electronics Eng. 34850, Avcilar, Istanbul, Turkey, E- \\ mail:akgundog@istanbul.edu.tr \\ ${ }^{2}$ Instiut PRISME / LESI, University of Orleans, 12, rue de Blois, 45067 Orléans Cedex 2, FRANCE \\ ${ }^{3}$ Equipe INSERM U658, Hospital of Orleans, 1, rue Porte Madeleine, 45000 Orléans, FRANCE
}

\begin{abstract}
In order to prevent bone fractures due to disease and ageing of the population, and to detect problems while still in their early stages, 3D bone micro architecture needs to be investigated and characterized. Here, we have developed various image processing and simulation techniques to investigate bone micro architecture and its mechanical stiffness. We have evaluated morphological, topological and mechanical bone features using artificial intelligence methods. A clinical study is carried out on 2 populations of arthritic and osteoporotic bone samples. The performances of Adaptive Neuro Fuzzy Inference System (ANFIS), Support Vector Machines (SVM) and Genetic Algorithm (GA) in classifying the different samples have been compared. Results show that the best separation success (100\%) is achieved with Genetic Algorithm.
\end{abstract}

Keywords: Trabecular bone, Hybrid Skeleton Graph Analysis, SVM, GA, Anfis.

\section{Introduction}

The interest in three-dimensional image analysis is increasing in different fields of image processing. In biomedical systems many new tomographic modalities now model various 3-D images of organs and bones. Applications of these methods range from biological studies [1] to character recognition [2]. Image analysis techniques are used in order to obtain morphological and topological characterizations. 
In the case of bone, the micro architecture plays an important role in osteoporosis, osteoarthritis and the prediction of fractures. To characterize such material, Cruz-Orive [3] and Levitz et al. [4] evaluated the mean size of materials by averaging 3D data. Odgaard et al. [5] and Vogel [6] defined a connectivity index that can be computed by scanning data and storing topological information. In addition to these methods, the morphological structure can be accessed by determining parameters that can be computed directly from the 3D image without an underlying model assumption [7, 8]. This work led however to global methods based on physical models that cannot give precise information about the medium's structure and its local properties. Nowadays, it is possible to study the object at a local scale and obtain information from each of the elements that compose the structure of the material [9]. A skeleton-based technique called Line Skeleton Graph Analysis (LSGA) [10] enables the bone micro architecture to be studied at a local scale, providing global information about the bone. This method preserves the topology of the medium but is not suitable for structures comprising different shapes such as trabecular bone, which is composed of rod-shaped and plate-shaped elements. In this case, all non-cylindrical shapes are better described by 2D-surfaces rather than by 1D-curves.

We have developed a new technique called Hybrid Skeleton Graph Analysis (HSGA) [11] to create structural models of disordered porous media. The method is based on [9], but takes into account the shape of the object, in order to improve the geometrical approximation of [9], since disordered porous media such as trabecular bone are not homogeneous but are composed of rodshaped and plate-shaped elements. Curve thinning and surface thinning are efficient respectively for one or the other, but not for both. Our early work for skeletonizing hybrid-shaped media focused on providing a tool for objects composed of rods and plates [10]. The HSGA method is based on a homotopic hybrid skeleton using curve and surface thinning techniques which preserve Betti-numbers [12]; the topology of the medium is thus perfectly preserved.

At the same time, the combination of skeletons and Finite Elements (FE) analysis to evaluate the stiffness of large-scale porous media has recently shown great potential [13, 14]. We have improved on a previously published beam Finite Element Analysis (FEA) in [13]. A full protocol $[15,16]$ has been developed for evaluating the stiffness of large scale porous media also based on the HSGA and using the advantage of both beam and shell Finite Elements to enable a fast and precise mechanical simulation. 
The improvement of these techniques has enabled the determination of various parameters for quantifying the morphology and the stiffness of the bone micro architecture. These parameters have to be combined efficiently to study bone diseases. For this purpose, knowledge modeling is achieved using Artificial Intelligence (AI) approaches which are developed by human expertise [17-20]. These models are based on training and testing. The Adaptive Neuro Fuzzy Inference System (ANFIS), Support Vector Machines (SVM) and Genetic Algorithm (GA) are the most successful tools for predicting input-output data set systems.

This work presents a study performed on 2 populations of trabecular bone samples with an a priori knowledge of fracture risk, 9 osteoarthritic (OA) samples and 9 osteoporotic (OP) samples. The different improved techniques have been processed on each of the 18 bone samples, producing features which are then used to quantify the microarchitectural bone quality. These parameters are used as entries for different artificial intelligence systems, namely: Adaptive Neuro-Fuzzy Inference System (ANFIS), Support Vector Machines (SVM) and Genetic Algorithm (GA). We also investigated whether the combination of these different techniques contributes to a better prediction of the nature (OA or OP) of each sample studied.

The paper is organized as follows: first, the different artificial intelligence systems used (ANFIS, SVM and GA) are presented. Then, the techniques used to compute the OA and OP data are presented. The results from the clinical study follow. A discussion concludes this work.

\section{Material and Methods}

This section presents the three artificial intelligence methods, Adaptive Neuro Fuzzy Inference System (ANFIS), Support Vector Machines (SVM) and Genetic Algorithm (GA), as well as the image processing techniques, Hybrid Skeleton Graph Analysis (HSGA) and Finite Element Analysis (FEA) used to classify each sample of the two studied populations (OA and OP).

\section{Artificial Analysis}

Artificial Intelligence (AI) is the area of computer science which deals with machine help in finding solutions to complex problems in a more human-like fashion. This generally involves borrowing characteristics from human intelligence, and applying them as algorithms in a computer- friendly way. 


\subsection{Adaptive Neuro-Fuzzy Inference System (ANFIS)}

ANFIS is a combination of ANN and Fuzzy Inference System (FIS) [18] which is used to determine the parameters of FIS. ANFIS implements a Takagi Sugeno Kang (TSK) [21] fuzzy inference system in which the conclusion of the fuzzy rule is constituted by a weighted linear combination of the crisp inputs.

\subsubsection{Architecture of ANFIS}

ANFIS is a fuzzy Sugeno model implemented in the framework of adaptive systems to facilitate learning and adaptation [17]. Such a framework makes ANFIS modeling more systematic and less reliant on expert knowledge. To present the ANFIS architecture, two fuzzy if-then rules based on a first order Sugeno model are considered [17, 18, 21, 22].

Rule 1: If $\left(\mathrm{x}\right.$ is $\left.\mathrm{A}_{1}\right)$ and $\left(\mathrm{y}\right.$ is $\left.\mathrm{B}_{1}\right)$ then $f_{1}=p_{1}+q_{1} y+r_{1}$,

Rule 2: If $\left(\mathrm{x}\right.$ is $\left.\mathrm{A}_{2}\right)$ and $\left(\mathrm{y}\right.$ is $\left.\mathrm{B}_{2}\right)$ then $f_{2}=p_{2}+q_{2} y+r_{2}$.

where $x$ and $y$ are the inputs, $A_{i}$ and $B_{i}$ are the fuzzy sets, $f_{i}$ are the outputs within the fuzzy region specified by the fuzzy rule, and $\mathrm{p}_{\mathrm{i}}, \mathrm{q}_{\mathrm{i}}$ and $\mathrm{r}_{\mathrm{i}}$ are the design parameters that are determined during the training process.

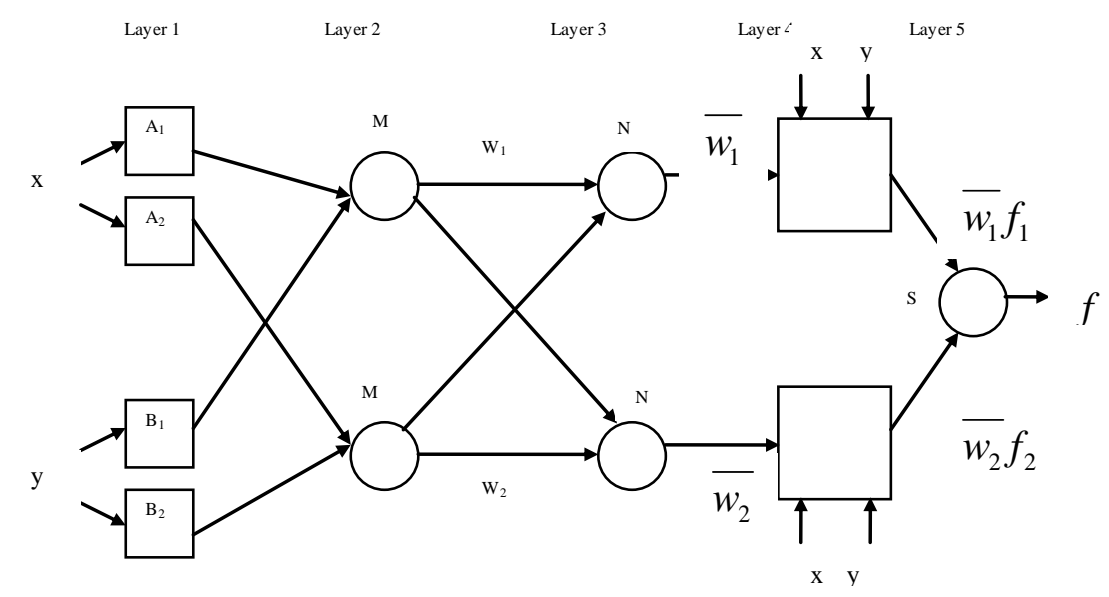

Figure 1. ANFIS architecture. 
The ANFIS architecture for the implementation of these two rules is shown in Figure 1, in which a circle indicates a fixed node, whereas a square indicates an adaptive node. In layer 1, all the nodes are adaptive nodes. The outputs of layer 1 are the fuzzy membership grade of the input, which are given by

$$
\begin{aligned}
& O_{i=}^{1} \mu_{A i}(x), \quad \mathrm{i}=1,2, \text { or } \\
& O_{i \mathrm{i}=}^{1} \mu_{B i-2}(y), \quad \mathrm{i}=3,4,
\end{aligned}
$$

where $\mu_{A i}(x), \mu_{B i-2}(y)$ can adopt any fuzzy membership function. We will use the Gaussian membership function given by:

$$
\mu_{A_{i}}(x)=e^{-\frac{1}{2}\left(\frac{x-c_{i}}{\sigma_{i}}\right)^{2}}
$$

Where $c$ represents the membership function's (Mfs) center and $\sigma$ determined the Mfs width. $c_{i}$ and $\sigma_{i}$ are parameters to be learnt. These are the premise parameters.

In layer 2, the nodes are fixed. They are labeled with $\mathrm{M}$, indicating that they behave as a simple multiplier. The outputs of this layer can be represented as

$$
O_{i}^{2}=w_{i}=\mu_{A_{i}}(x) \mu_{B_{i}}(y), i=1,2
$$

which are the so-called firing strengths of the rules.

In layer 3, the nodes are also fixed nodes. They are labeled with $\mathrm{N}$, indicating that they play a normalization role to the firing strengths from the previous layer. The outputs of this layer can be represented as

$$
O_{i}^{3}=\bar{w}_{i}=\frac{w_{i}}{w_{1}+w_{2}}, i=1,2
$$

These are the so-called normalized firing strengths.

In layer 4 , the nodes are adaptive nodes. The output of each node in this layer is simply the product of the normalized firing strength and a first-order Sugeno model. Thus, the outputs of this layer are given by

$$
O_{i}^{4}=\bar{w}_{i} f_{i}=\bar{w}_{i}\left(p_{i} x+q_{i} y+r_{i}\right), i=1,2
$$


In layer 5, there is only one single fixed node labeled with $\mathrm{S}$. This node performs the summation of all incoming signals. Hence, the overall output of the model is given by

$$
O_{i}^{5}=\sum_{i=1}^{2} \overline{w_{i}} f_{i}=\frac{\left(\sum_{i=1}^{2} w_{i} f_{i}\right)}{w_{1}+w_{2}}
$$

It can be observed that there are two adaptive layers in this ANFIS architecture, namely the first layer and the fourth layer. In the first layer, there are three modifiable parameters $\left\{a_{i}, b_{i}, c_{i}\right\}$, which are related to the input membership functions. These parameters are the so-called premise parameters.

In the fourth layer, there are also three modifiable parameters $\left\{p_{i}, q_{i}, r_{i}\right\}$, pertaining to the first order Sugeno model. These parameters are called consequent parameters [22, 23].

\subsubsection{Hybrid Learning Algorithm of ANFIS}

The task of the learning algorithm for this architecture is to tune all the modifiable parameters, namely $\left\{a_{i}, b_{i}, c_{i}\right\}$ and $\left\{p_{i}, q_{i}, r_{i}\right\}$, in order to make the ANFIS output match with the training data. When the premise parameters $a_{i}, b_{i}, c_{i}$ of the membership function are fixed, the output of the ANFIS model can be written as:

$$
\begin{aligned}
& f=\frac{w_{1}}{w_{1}+w_{2}} f_{1}+\frac{w_{2}}{w_{1}+w_{2}} f_{2} \\
& f=\bar{w}_{1} f_{1}+\bar{w}_{2} f_{2} . \\
& f=\bar{w}_{1}\left(p_{1} x+q_{1} y+r_{1}\right)+\bar{w}_{2}\left(p_{2} x+q_{2} y+r_{2}\right) . \\
& f=\left(\bar{w}_{1} x\right) p_{1}+\left(\bar{w}_{1} y\right) q_{1}+\left(\bar{w}_{1}\right) r_{1}+\left(\bar{w}_{2} x\right) p_{2}+\left(\bar{w}_{2} y\right) q_{2}+\left(\bar{w}_{2}\right) r_{2} .
\end{aligned}
$$

Where $p_{1}, q_{1}, r_{1}, p_{2}, q_{2}, r_{2}$ are linear consequent parameters. When the premise parameters are not fixed, the search space becomes larger and the convergence of the training becomes slower. A hybrid algorithm combining the least squares method and the gradient descent method is adopted to solve this problem [24]. The hybrid algorithm is composed of forward pass and backward pass. The least squares method (forward pass) is used to optimize the consequent parameters with the 
fixed premise parameters. $[25,26]$. Once the optimal consequent parameters have been found, the backward pass starts immediately. The gradient descent method (backward pass) is used to optimize the premise parameters corresponding to the fuzzy sets in the input domain. The output of the ANFIS is calculated by employing the consequent parameters found in the forward pass. The output error is used to adapt the premise parameters by means of a standard back propagation algorithm. It has been proven that this hybrid algorithm is highly efficient in training the ANFIS $[26,27]$.

\subsection{Support Vector Machines (SVM)}

Support Vector Machine is an effective technique for data classification. The SVM proposed by Vapnik [19] has been extensively studied for classification, regression and density estimation. A classification task usually involves training and testing data which consist of some data instances. Each sample in the training set contains one "target value" (class labels) and several "attributes" (features). The goal of SVM is to produce a model which predicts the target value of data instances in the testing set where only the attributes are known. Figure 2 illustrates the map of input - output SVM space.

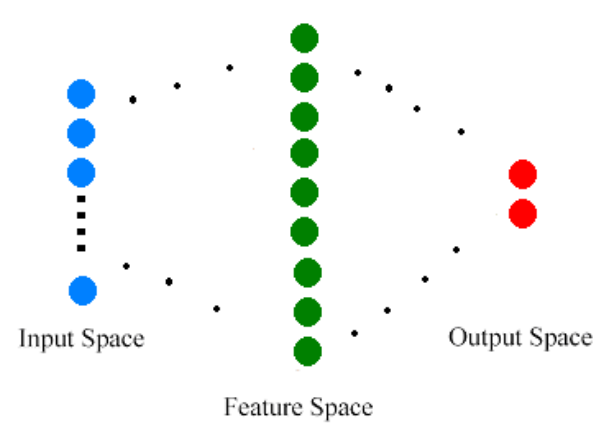

Figure 2. Mapping the Input and Output space.

In Figure 3, there are many possible linear classifiers that can separate the data, but there is only one that maximizes the margin (maximizes the distance between the nearest data point of each class). This linear classifier is termed the optimal separating hyper plane. 


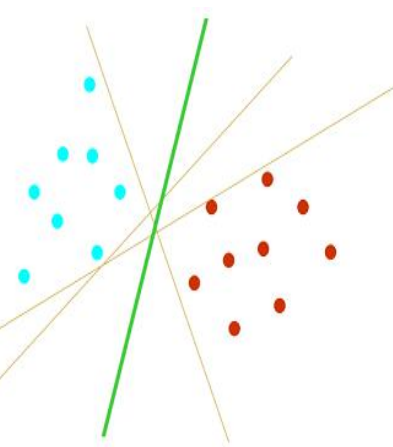

Figure 3. General separation of optimal hyper plane.

Based on the principle of structural risk minimization, the SVM upper bounds the expected risk (mean error rate measured on the test set) using the sum of the empirical risk (mean error rate measured on the training set) and a bound, called the Vapnik-Chervonenkis (VC) confidence. In order to construct an optimal decision hypothesis, the SVM holds the empirical risk fixed and minimizes the $\mathrm{VC}$ confidence by limiting the flexibility of the set of candidate functions searched by the machine (classifier). The minimum of this upper bound is reached by maximizing the margin between the decision hypothesis and the classes as defined using support vectors, thereby enabling the improvement of the classifier generalization ability.

Support vector machines (SVMs) are a set of related supervised learning methods used for classification and regression. They belong to a family of generalized linear classifiers. A special property of SVMs is that they simultaneously minimize the empirical classification error and maximize the geometric margin; hence they are also known as maximum margin classifiers. Two-dimensional space that separates two classes of data shows a hyper plane is depicted in figure 4. 


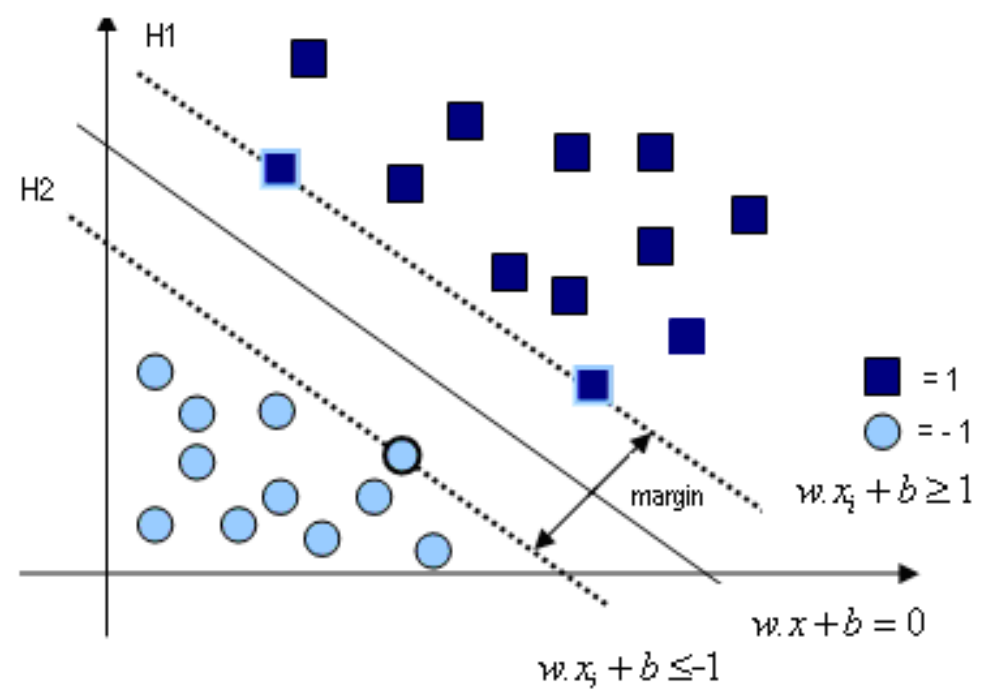

Figure 4. Linear classifier and margins.

$$
\begin{aligned}
& \mathrm{w}^{\mathrm{T}} \mathrm{x}_{\mathrm{i}}+\mathrm{b} \geq 1, \quad \mathrm{y}_{\mathrm{i}}=1 \\
& \mathrm{w}^{\mathrm{T}} \mathrm{x}_{\mathrm{i}}+\mathrm{b} \leq-1, \mathrm{y}_{\mathrm{i}}=-1
\end{aligned}
$$

The points on the planes $\mathrm{H} 1$ and $\mathrm{H} 2$ are the support vectors. A linear classifier is defined by a hyper plane's normal vector $w$ and an offset $b$. The decision boundary is, $w^{T} x_{i}+b=0$. Each of the two half spaces defined by this hyper plane corresponds to one class, $y_{i}=\operatorname{sgn}\left(w^{T} x_{i}+b\right)$ The margin of a linear classifier is the minimal distance of any training point to the hyper plane.

Viewing the input data as two sets of vectors in an n-dimensional space, an SVM will construct a separating hyper plane in that space, which maximizes the "margin" between the two data sets. To calculate the margin, we construct two parallel hyper planes, one on each side of the separating one, which are "pushed up against" the two data sets. Intuitively, a good separation is achieved by the hyper plane that has the largest distance from the neighboring data points of both classes. It is expected that the larger the margin or distance between these parallel hyper planes, the better the generalization error of the classifier will be.

Let $\left\{x_{i}, y_{i}\right\}, i=1,2, \ldots . . k, y_{i} \in\{-1,1\}$ and $x_{i} \in I R^{n}$ be the training samples where the training vector. $x_{i}$ and $y_{i}$ are corresponding target value. On input pattern $x$, the decision function of binary classifier is;

$$
f(x)=\operatorname{sign}\left(\sum_{i=1}^{k} y_{i} \alpha_{i} K\left(x, x_{i}\right)+b\right)
$$




$$
\operatorname{sgn}(u)=\left\{\begin{array}{lll}
1 & \text { for } & u>0 \\
-1 & \text { for } & u<0
\end{array}\right.
$$

where $\mathrm{k}$ is the number of learning patterns, $y_{i}$ is the target value of learning pattern $x_{i}, \mathrm{~b}$ is a bias, and $K\left(x, x_{i}\right)$ is a kernel function which high-dimensional feature space.

The idea of the kernel function is to enable operations to be performed in the input space rather than the potentially high dimensional feature space. Hence the inner product does not need to be evaluated in the feature space. This provides a way of addressing the problem of dimensionality. However, the computation is still critically dependent upon the number of training patterns and providing a good data distribution for a high dimensional problem will generally require a large training set. This theory is based upon Reproducing Kernel Hilbert Spaces (RKHS) [28-30]. An inner product in feature space has an equivalent kernel in input space, $K\left(x, x^{\prime}\right)=<\Phi(x), \Phi\left(x^{\prime}\right)>$

provided certain conditions hold. If $\mathrm{K}$ is a symmetric positive definite function, which satisfies Mercer's Conditions,

$$
\begin{array}{ll}
K\left(x, x^{\prime}\right)=\sum_{m}^{\infty} a_{m} \Phi_{m}(x), \Phi_{m}\left(x^{\prime}\right), & a_{m} \geq 0 \\
\iint K\left(x, x^{\prime}\right) g(x) g\left(x^{\prime}\right) d x d x^{\prime}>0, & g \in L_{2},
\end{array}
$$

then the kernel represents a legitimate inner product in feature space. Valid functions that satisfy Mercer's conditions are now given, which unless stated are valid for all real x and x'. A polynomial mapping is a popular method for non-linear modeling as,

Polynomial (homogeneous): $k\left(x, x^{\prime}\right)=\left(x . x^{\prime}\right)^{d}$

Polynomial (inhomogeneous): $k\left(x, x^{\prime}\right)=\left(x \cdot x^{\prime}+1\right)^{d}$

Radial basis functions have received significant attention, most commonly with a Gaussian form:

$k\left(x, x^{\prime}\right)=\exp \left(-\frac{\left\|x-x^{\prime}\right\|^{2}}{2 \sigma^{2}}\right)$

Classical techniques utilizing radialbasis functions employ some method of determining a subset of centers. Typically a method of clustering is first employed to select a subset of centers. An 
attractive feature of the SVM is that this selection is implicit, with each support vector contributing one local Gaussian functions, centered at that data point.

\subsection{Genetic Algorithm (GA)}

This method was inspired by work first reported by Siedlecki and Sklansky [20]. The genetic algorithm is a method for solving both constrained and unconstrained optimization problems that is based on natural selection, the process that drives biological evolution. In recent years, the genetic algorithms are used in many medical applications [31].

The genetic algorithm repeatedly modifies a population of individual solutions. At each step, the genetic algorithm selects individuals at random from the current population to be parents and uses them to produce the children for the next generation. Over successive generations, the population "evolves" toward an optimal solution. Genetic algorithms can be applied to solve a variety of optimization problems that are not well suited for standard optimization algorithms, including problems in which the objective function is discontinuous, non differentiable, stochastic, or highly nonlinear.

The genetic algorithm uses three main types of rules at each step to create the next generation from the current population. Firstly, selection rules select the individuals, called parents that contribute to the population at the next generation. Secondly, crossover rules combine two parents to form children for the next generation. Finally, mutation rules apply random changes to individual parents to form children. GA is designed to simulate the evolutionary processes that occur in nature $[32,33]$.

The basic idea is derived from the Darwinian theory of survival of the fittest. Three fundamental mechanisms drive the evolutionary process: selection, crossover and mutation within chromosomes. As in nature, each mechanism occurs with a certain probability, allowing for some randomness. Selection occurs on the current population by choosing the most-fit individuals to reproduce. Reproduction, then, can result in the crossover and/or mutation of parent genes to form new solutions. The ratio of heuristic and stochastic decisions, at best, creates a natural balance between survival and evolution.

For each generation of the GA, individual solutions are evaluated using a fitness function. The evaluation method is a crucial component of the selection process since offspring for the next 
generation are determined by the fitness values of the present population. Figure 5 provides a simple diagram of the iterative nature of genetic algorithms. The generational process ends when the user-defined goal is reached. In most cases, the number of generations is a constant and is set by the user [34].

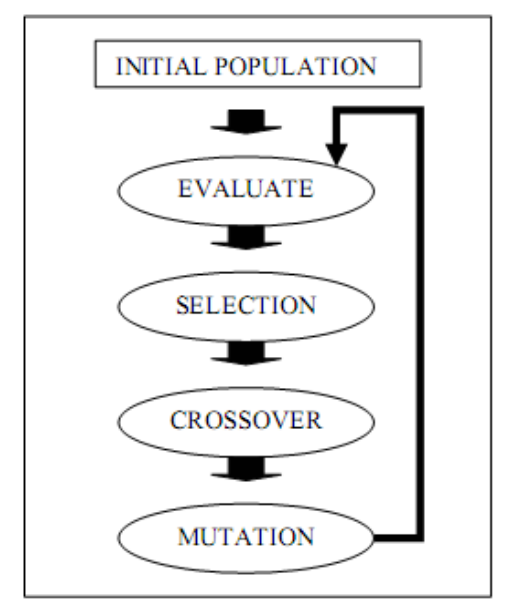

Figure 5. Flow diagram depicting the evolutionary process of genetic algorithm.

The algorithm is;

1. Population is randomly initialized

2. Fitness of population is determined.

3. Repeating...

a) Select parents from population.

b) Perform crossover on parents creating population.

c) Perform mutation of population.

d) Determine fitness of population

4. until best separately is good enough.

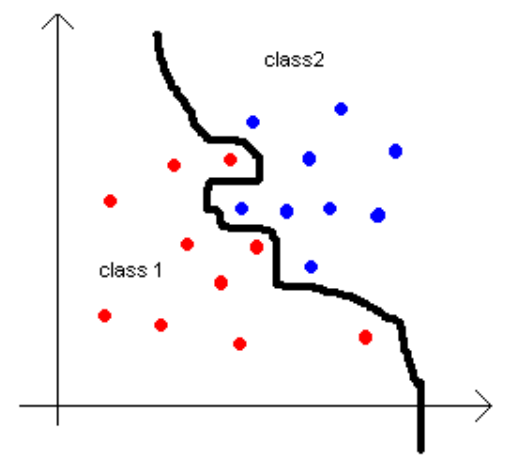

Figure 6. General separation of two classes by GA. 
A classification problem deals with associating a given input pattern with one of the distinct classes. Patterns are specified by a number or some measurements so it is natural to think of them as $d$-dimensional vectors, where $d$ is the number of different features. This representation gives rise to a concept of feature space. The classification problem determines which of the regions a given pattern falls into. If classes do not overlap they are said to be separable and, in principle, one can design a decision rule which will successfully classify any input pattern. A decision rule determines a decision boundary which partitions the feature space into regions associated with each class. It represents the best solution to the classification problem. Figure 6 illustrates a 2dimensional feature space with two classes occupying regions of the space. This method carries out feature extraction and classifier design simultaneously, through genetic learning and evolution as shown in figure 7.

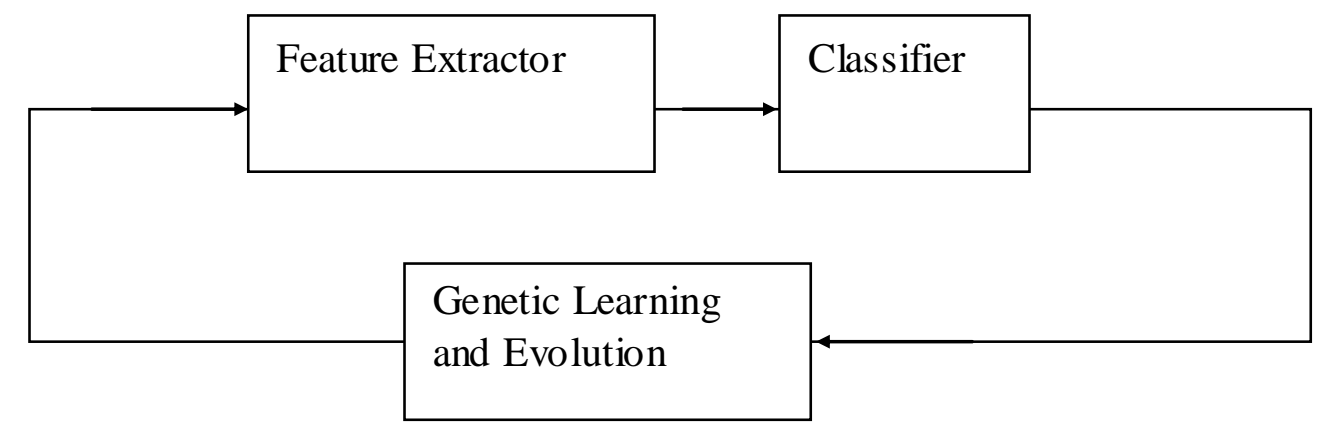

Figure 7. Feature extractor and classifier with feedback learning system.

The aim is to design a decision rule which is easy to compute and yields the smallest possible probability of misclassification of input patterns from the feature space.

\section{Image Processing and Mechanical Assessment}

The next section details the techniques developed to investigate bone micro architecture and its mechanical stiffness.

\subsection{Hybrid Skeleton Graph Analysis (HSGA)}

The Hybrid Skeleton Graph Analysis (HSGA) [11] model is a high-level representation of a hybrid shaped porous medium composed of rods and plates such as trabecular bone. The morphology and topology of disordered porous media were first investigated using global methods based on physical models that cannot give complete information about the trabeculae's 
structure and its local properties. In 2000 a new method called Line Skeleton Graph Analysis (LSGA) [10] was introduced for studying porous media using a skeleton-based technique. However, as it uses a curve skeleton, the LSGA has its drawbacks since all non-cylindrical shapes are better described by $2 \mathrm{D}$-surfaces rather than by $1 \mathrm{D}$-curves.

\section{a) Hybrid skeleton}

The HSGA [11] relies on a new hybrid skeletonization process which is computed by processing curve or surface thinning, depending on the local shape of the object. To switch between the 2 skeleton variants, the HSGA improves a recent algorithm [/***compléter bonnassie] which classifies the voxels of an object according to their topological predisposition to belong to a plate or to a rod zone [36]. Then, surface and curve thinning algorithms are applied depending on whether each primitive belongs to the set of rods or plates. The proposed hybrid thinning algorithm preserves connectivity which is an essential feature when characterizing porous media such as trabecular bone. Figure 8(a) and figure 8(b) show respectively an extracted portion of a trabecular bone sample and its hybrid skeleton.

\section{b) Classification}

Once the hybrid skeleton (figure 8(a)) has been computed, a classification step is applied to label each voxel of the skeleton according to its structural role. The classification consists in affecting a class identifier to each voxel of the skeleton according to its structural role. We define 4 classes of voxels: "rod", "line-end", "plate" and "node". As in [10], 2 voxels of the solid phase are neighbors if they are 26-connex (i.e. they share at least one corner). Similarly, voxels of the pore phase are neighbors if they are 6-connex (i.e. they share at least a face).

The voxel classification is based on a 3-step process:

1 Step 1: Initialization

All voxels are set to "plate".

2 Step 2: Determination of rod-shaped 1D-curves

A voxel is marked as "rod" if it has only 2 solid neighbors that are not neighbors themselves. A voxel is marked as "line-end" if it has only 1 neighbor.

3 Step 3: Determination of interfaces between elements

Any voxel is set to node if: 
- It has more than 2 "rod" or "line-end" neighbors

- It is a "plate" and has a "rod" or "line-end" neighbor

Figure 8(c) shows the result of the classification step. Each voxel of the skeleton has been marked as belonging to a "rod", "line-end", "plate" or "node" class.

\section{c) Individualization}

After classification, the role of each voxel in the skeleton can be determined. However, the plates and rods of the structure cannot be processed one by one, since they have not been extracted and individualized. In each class, the structure elements have to be marked separately. To do so, all the information associated to one plate or rod must be collected. This is the object of the individualization process which processes recursively all the voxels of the structure and determines the interfaces of each element of the object. The elements individualization algorithm is implemented as a 3 -step loop:

$4 \quad$ Step 1: Finding a solid phase element

An initial voxel (the seed) is chosen in the classified skeleton. It can be of type "plate" for a plate element or "rod" for a rod element.

$5 \quad$ Step 2: Spreading the information

The seed is recursively spread through the skeleton voxels until the element boundaries are found. Boundary voxels can be of type "node" or "line-end".

6 Step 3: Registering the new element

Once an element has been entirely defined, its voxels and interfaces are registered in the model.

this information is registered in the HS GA model. Figure 8(c) illustrates how different elements of the structure are individualized.

\section{d) Segmentation}

The classified and individualized skeleton supports a standalone use as it already contains information about the medium. The analysis can be improved however by processing segmentation of the original object. For this purpose, we implement a 3D region-growth iterative 
process. It takes the skeleton voxels of each element as a seed and iteratively merges neighbors from the original volume. As a result, each solid voxel of the object is finally associated to an element of the HSGA model (plate or rod). Figure 8(d) illustrates this segmentation. All the voxels of the original object have been referenced in the model, and the global shape is unchanged. It can be seen that these voxels are associated to different plate or rod elements (shown by different colors), according to the natural region growth to which they belong.

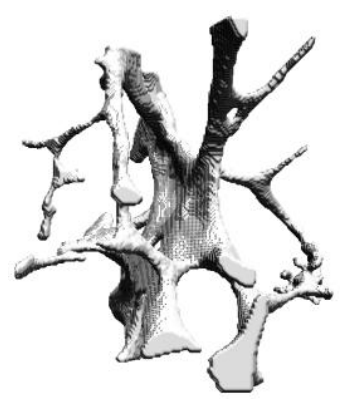

(a)

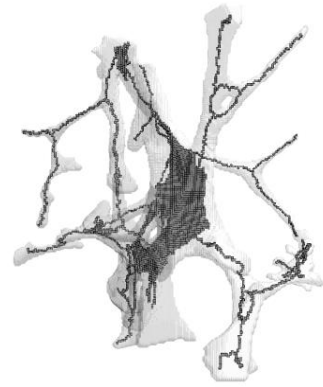

(b)

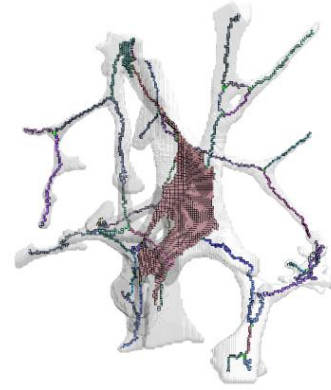

(c)

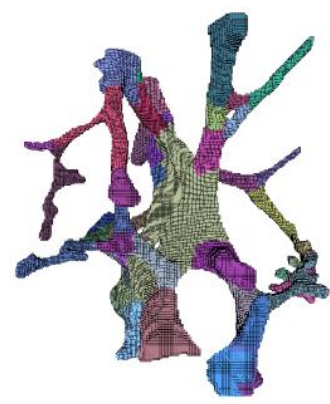

(d)

Figure 8. Illustration of the HSGA model and its different computing steps on a trabecular bone sample. Original trabecular bone (a), hybrid skeleton (b), classified hybrid skeleton (c), final HSGA segmented model (d). In (b) and (c), the skeleton is stacked over the original object.

Once the HSGA model has been completed, a template of the trabecular network is obtained. Each vertex and branch is localized, enabling the extraction of each trabecula from the network. The HSGA model contains morphological, topological and volumetric information and many parameters can be measured which are discussed in the clinical study. In the next section, The HSGA is used as a basis for the generation of finite element models.

\subsection{Mechanical Assessment}

The reference for Finite Elements (FE) analysis of discrete samples is unquestionably voxel-toelement conversion as discus sed in many articles $[37,38]$. However, in the case of skeleton-based models, other types of elements can be used to simplify large-scale problems and prevent time resource consumption. In this section, we explain our modeling choices, relative to the compromise between simplification and loss of accuracy in the simulation. First, the method used 
to convert rod shapes to beam chains is explained. Then the triangulation technique for converting plate shapes to shell elements is described.

\section{a) Rods to beam elements chains conversion}

The FE that matches the geometry of a straight rod is the beam element. Its geometry is described as a 1D segment, which is assigned a circular cross section. This technique was first investigated in [13] to assess the stiffness of trabecular bone. However, results show that modeling the bone by a simple rod network is not sufficient to obtain an accurate assessment of stiffness, due to geometrical lack of accuracy. Lenthe et al. [14] explored this field of beam-modeling rod shapes in porous media, but did not resolve the shape accuracy issues.

In order to convert a rod item to FE, we introduced the "beam chains" concept [15]. Based on a feature extraction technique used in the field of 3D animation [39], the beam chain introduces evenly set intermediate nodes on the curve skeleton as seen in figure 9. This process is called "splitting" as it breaks the curve into small segments that better match the curvature of the rod item. Finally, each split element (i.e. each effective beam FE) is assigned a section according to the local thickness values recorded in the HSGA model, as illustrated in figure 9.

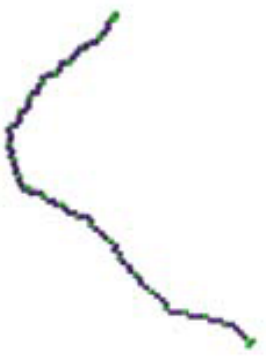

(a)

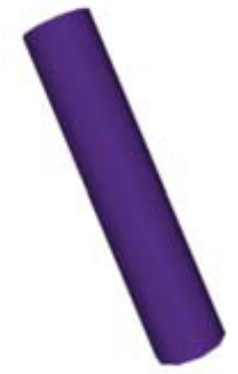

(b)

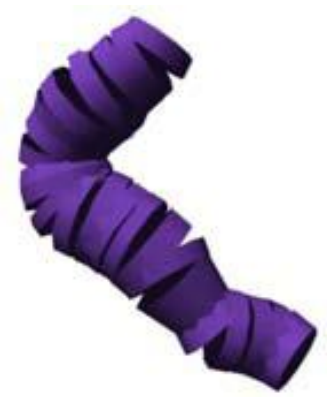

(c)

Figure 9. Illustration of the rods to beam elements chains conversion: voxels of the 1D path extracted from its curve skeleton with intermediate nodes (a), simple cylinder model assumption using the rod's volume and skeleton length to compute its section as $S=V / 1(b)$, and beam chain modeling of the item using local thicknesses (c)

\section{b) Plates to shell elements conversion}

Plate zones are badly described in the case of beam-only models $[15,36]$, which lead to a nonnegligible bias for morphological results [13]. It is suspected that this lack of geometrical 
accuracy also alters mechanical results. In fact, modeling plates is much more challenging than modeling rods, since research in this area is scanty. Recently, Lenthe et al. [14] proposed converting a plate into a set of beams instead of a single beam. Yet, the efficiency of this conversion is questionable since the notion of beam set is not geometrically obvious, and the beam sections are model-dependent. We present here an original approach that combines the power of a new triangulation method and a better choice of FE type to improve plate modeling. The FE used to describe planar shapes is the shell element. It is defined as a 2D medial surface geometry (either triangle or quad strips for example), on which each element is assigned a thickness value. The challenge in this case was to be able to transform the 26-connected plate voxels sets into a list of simple 2D primitives such as triangles or quads. Meshing iso-surfaces has been widely explored in computer graphics since the early 80 s, leading to a great range of techniques: surface fitting, surface tracking (also known as continuation methods) and spatial sampling. However, the case of crossing and stacking manifold surfaces from disordered data is still difficult to handle with criteria like Delaunay tracking, and none of these techniques suited our needs. We chose therefore to draw on a well-known spatial sampling method: Marching Cubes (MC) [40], which subdivides space into cells and searches those that intersect the implicit surface. Our algorithm, called Surface Marching Cubes (SMC), computes the full-resolution triangulation of any 26-connected surface by following the MC principle while using a new set of neighborhood patterns. All the triangles linking the voxels of the surface are generated. Figure 10 presents the result of the triangulation of a simple 26-connex surface set. Finally, each shell element (i.e. each triangle) is assigned a section according to the local thickness value in the thickness map, as was done for the beam elements in the case of rod shapes.

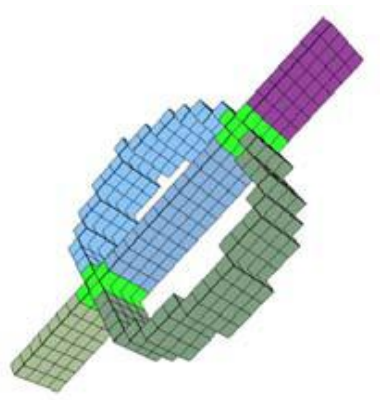

(a)

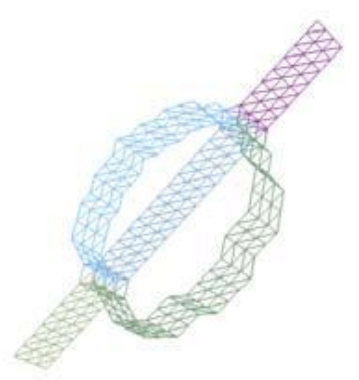

(b) 
Figure 10. Illustration of the plates to shell elements conversion: 26 -connex voxels of the 2D surface extracted from its surface skeleton (a), and example of a shell elements triangulation using Surface Marching Cubes (b).

The HSGA is used as a basis for the generation of FE models. Thickness map matching [41] is processed to improve the geometrical accuracy of all the FE models generated, using local cross-section values.

All the FE models were imported into the commercial software "Abaqus", which was used to estimate their apparent Young's Modulus. As the simulation required material definition, we assigned the entire model the same behavior, with a bone tissue characterized by an arbitrary Young's modulus of $15 \mathrm{GPa}$ and a Poisson's coefficient of 0.3 . These values are close to those found in the literature $[14,42]$. In this kind of comparative study, the material's behavior does not really matter since the measured reaction forces are compared relatively. Once defined, each model was tested in compression in the 3 space directions ( $\mathrm{x}, \mathrm{y}$ and $\mathrm{z}$ ). Boundary conditions on the cube's faces were set to zero for translations perpendicular to the faces and their complementary rotations. Complementary translations were also blocked for the 2 faces perpendicular to the compression axis. Then, a displacement value $\Delta l$ was applied on the compression direction's front face. A small displacement of $2 \%$ of the cube's size (i.e. 0.0913 $\mathrm{mm}$ ) avoided non-linearity issues. The apparent Young's modulus (Eapp) of the model was then computed [14] as:

$$
\text { Eapp }=\left(\sum R F / l^{2}\right) /(\Delta l / l)
$$

where $\Sigma R F$ represents the measured sum of the Reaction Forces (RF) on each node of the compression face, and 1 is the size of the cube's side.

\section{Results}

The HSGA is an efficient tool for the analysis of porous media. This section presents its usefulness to describe trabecular bone samples from a clinical study. For this purpose, the medical staff at the hospital of Orleans (France) provided us with 2 populations of 9 samples each, extracted from post-mortem femoral head and acquired using a Sky Scan micro-scanner 
with a high-resolution $\mu$-CT $(12 \mu \mathrm{m})$. The first 9 samples came from OsteoArthritic (OA) patients, whose bone structure is known to be hypertrophied, increasing the bone density. The other 9 samples were extracted from OsteoPorotic (OP) patients characterized by the deterioration of bone micro architecture which leads to bone fragility and fracture risk. As the characteristics of the 2 populations are previously known, they can be used to verify the separating power of any feature that is said to reflect the bone micro architecture alterations. The numerical samples are $400^{3}$ isotropic voxels 8-bit grey level volumes. They were pre-processed as follows: after applying a median filter, they were binarized at the local minimum threshold between the 2 modes of their histogram. The Hoshen-Kopelman [43] clustering algorithm was then used to remove non-connected solid voxel sets, as there can be no isolated material in the bone sample. Figure 11 shows 2 extracts from an OA and an OP sample. It can be seen that there is more solid material in the OA sample.

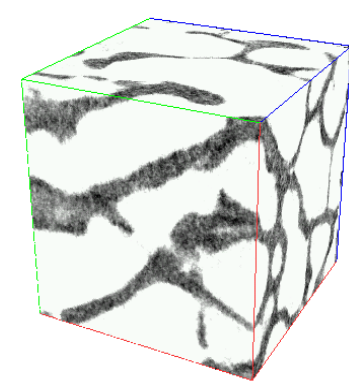

$(\mathrm{OA})$

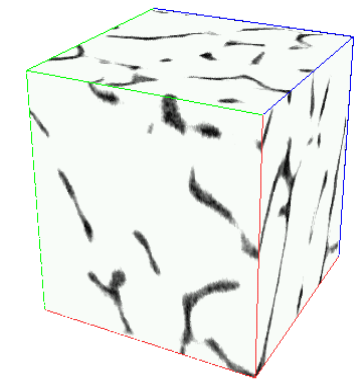

(OP)

Figure 11. Two $64^{3}$ voxels extracts from Osteoarthritis (OA) and Osteoporotic (OP) samples to illustrate the micro architectural differences in the two trabecular bones. The solid phase (bone) is illustrated in black while the pore phase is in white.

Using the Hybrid Skeleton Graph Analysis, topological, morphological and mechanical features can be measured as described below.

\section{a) Topological parameters}

As the HSGA conserves the connectivity of the studied media, it is possible to measure topological parameters such as: Connectivity (Beta1), Number of cavities of the solid phase (Beta2), Connectivity Density (Conn.D, $\mathrm{mm}^{-3}$ ). 


\section{b) Morphological parameters}

From a morphological point of view, the HSGA enables the following non exhaustive list of parameters to be measured: Bone Mineral Density (BMD, defined as Bone Volume over Total Volume, BV/TV), Bone Surface over Total Volume (BS/TV, $\mathrm{mm}^{-1}$ ), Bone Surface over Bone Volume (BS/BV, $\mathrm{mm}^{-1}$ ), Rod Volume (Rod.V, $\mathrm{mm}^{3}$ ), Rod Proportion (Rod.Prop, \% ), Plate Volume (Plate.V, $\mathrm{mm}^{3}$ ), Plate Proportion (Plate.Prop, \%), Element Number (El.N defined as Ro.N plus Pl.N), Plate Number (Pl.N), Rod Number (Ro.N), Rod Length (Ro.L, mm), Rod Volume (Ro.V, $\mathrm{mm}^{3}$ ), Rod Section (Ro.S, $\mathrm{mm}^{2}$ ), Rod Thickness (Ro.Th, mm), Plate Surface (Pl.S, mm²), Plate Volume (Pl.V, mm ${ }^{3)}$, Plate Thickness (Pl.Th, mm).

\section{c) Mechanical parameters}

Finally, associating the HSGA model to Finite Element Analysis allows measurement of new features such as: Mesh Shell Number (M.Sh.N), Mesh Node Number (M.No.N), Mesh Beam Number (M.Be.N), and Young's Modulus ( $\left.\mathrm{E}_{\mathrm{App}}(\mathrm{x}, \mathrm{y}, \mathrm{z})\right)$ in the 3 Space directions ( $\mathrm{x}, \mathrm{x}$ and $\left.\mathrm{z}\right)$.

Table 1 gives all the input parameters with minimum, maximum, mean and standard deviation values for each of the 27 parameters described above. Figure 12 shows all the input parameters' distribution versus one output parameter value. 
Table 1: Input parameters obtained from HSGA and FE Analysis.

\begin{tabular}{|c|c|c|c|c|c|}
\hline & Parameters & Minimum & Maximum & Mean & StdDev \\
\hline 1 & Beta1 & 259 & 1650 & 795,111 & 336,375 \\
\hline 2 & Beta2 & 3 & 41 & 20,33 & 12,654 \\
\hline 3 & Conn.D & $-14,692$ & $-2,294$ & $-6,962$ & 2,971 \\
\hline 4 & $\mathrm{BV} / \mathrm{TV}$ & 0,058 & 0,321 & 0,209 & 0,067 \\
\hline 5 & MC.BS/TV & 1,418 & 3,946 & 3,194 & 0,634 \\
\hline 6 & MC.BS/BV & 11,326 & 24,325 & 16,16 & 3,28 \\
\hline 7 & Rod.V & 3,469 & 13,939 & 8,791 & 2,578 \\
\hline 8 & Rod.Prop & 0,193 & 0,881 & 0,405 & 0,147 \\
\hline 9 & Plate. V & 0,773 & 27,282 & 14,492 & 6,097 \\
\hline 10 & Plate.Prop & 0,119 & 0,807 & 0,595 & 0,147 \\
\hline 11 & Shape. Ratio & 0,24 & 7,379 & 1,012 & 1,606 \\
\hline 12 & El.N & 604 & 3414 & $1,868,611$ & 822,669 \\
\hline 13 & Ro.N & 445 & 2438 & $1,448,778$ & 589,738 \\
\hline 14 & Pl.N & 37 & 976 & 419,833 & 253,171 \\
\hline 15 & Ro.L & 0,174 & 0,444 & 0,245 & 0,065 \\
\hline 16 & Ro.V & 0,002 & 0,009 & 0,004 & 0,002 \\
\hline 17 & Ro.S & 0,017 & 0,073 & 0,039 & 0,016 \\
\hline 18 & Ro.Th & 0,149 & 0,305 & 0,218 & 0,044 \\
\hline 19 & Pl.S & 0,069 & 0,616 & 0,243 & 0,132 \\
\hline 20 & Pl.V & 0,022 & 0,101 & 0,05 & 0,019 \\
\hline 21 & Pl.Th & 0,163 & 0,361 & 0,266 & 0,054 \\
\hline 22 & Mesh.Sh.N & 15376 & 338683 & 211581,39 & $74,493,854$ \\
\hline 23 & Mesh.No.N & 10292 & 186793 & 117405,94 & $40,368,399$ \\
\hline 24 & Mesh.Be.N & 791 & 3549 & $2,253,722$ & 650,924 \\
\hline 25 & Mec.Eapp.X & 3,405 & 265,664 & 72,669 & 60,356 \\
\hline 26 & Мec.Eapp. Y & 4,856 & 358,696 & 99,122 & 97,839 \\
\hline 27 & Mec.Eapp.Z & 11,126 & 164,913 & 76,068 & 43,687 \\
\hline
\end{tabular}




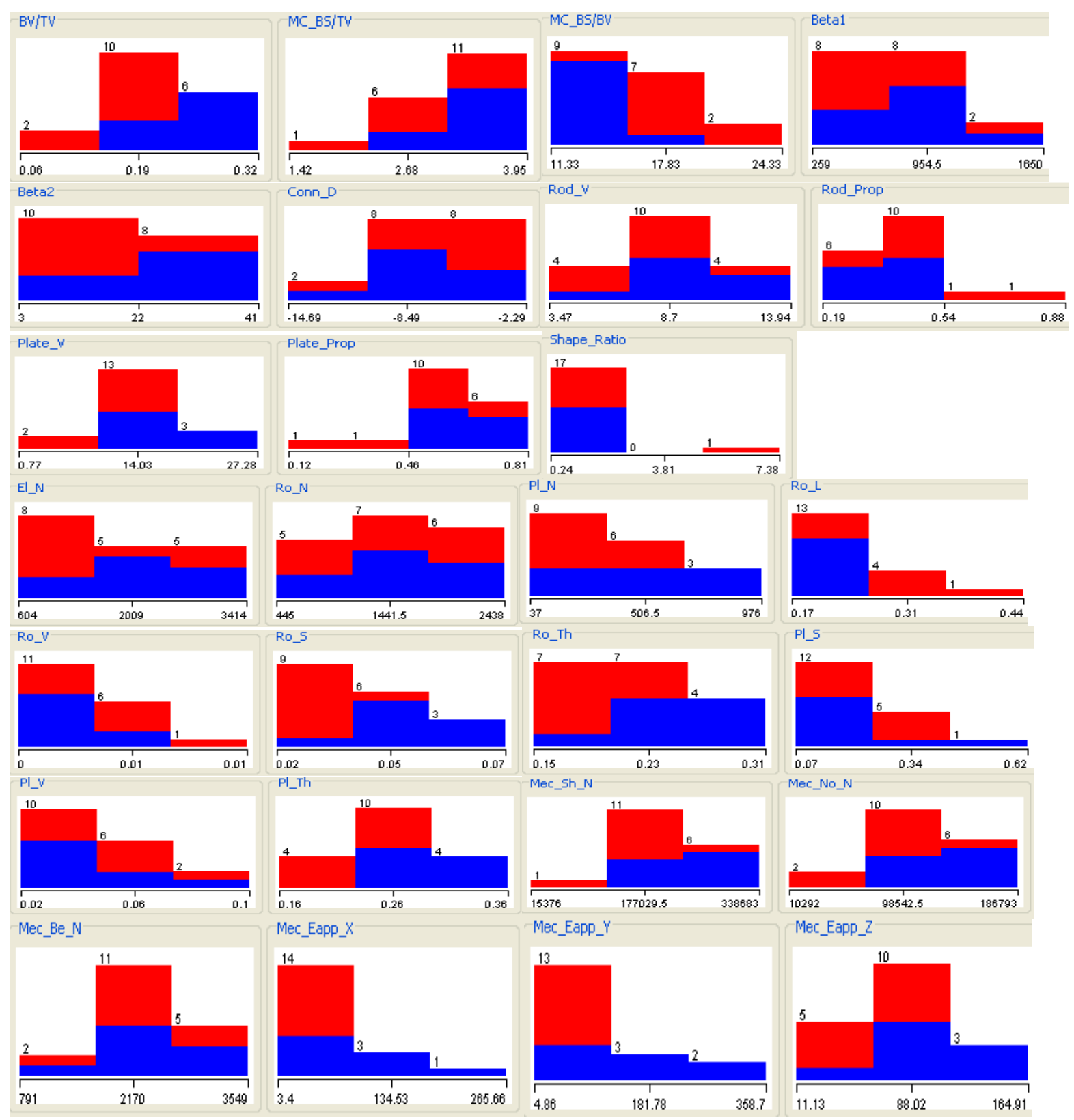

a)

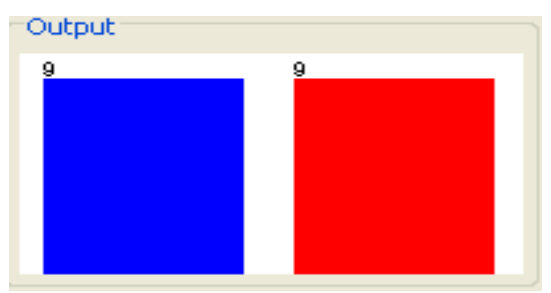

b)

Figure 12. Input and output parameters. a) Distribution of 27 Input parameters versus 1 Output Parameter, b) One output Parameter of two classes (OA, OP). 
Figure 12 shows that none of the computed 27 parameters is able to distinguish significantly between the 2 populations studied. This study involves 18 samples with 27 input parameters and 1 output parameter. 12 samples are used for prediction and 6 for the te st to be classified as OA or OP with Adaptive Neuro-Fuzzy Inference System (ANFIS), Support Vector Machines (SVM) and Genetic Algorithm (GA). Firstly we used ANFIS (Figure 13).

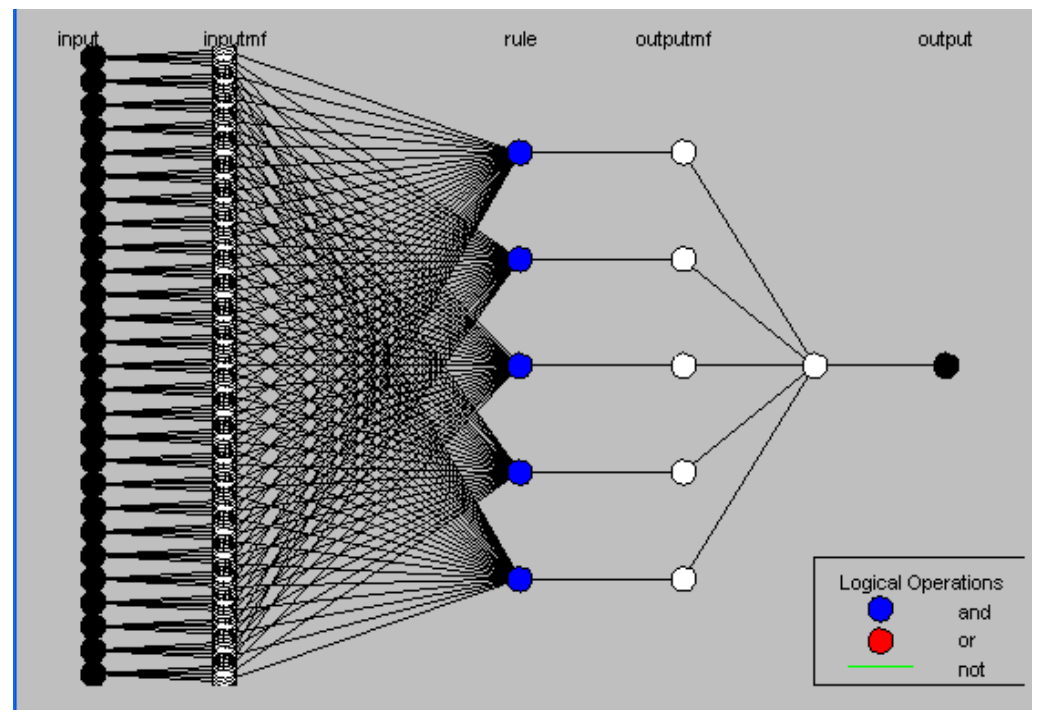

Figure13. Architecture of our ANFIS model: 27 inputs and 1 output with 4 rules.

In ANFIS model, Gaussian Membership Function and 5 rules were used. Therefore, 18 samples are not enough for accurate prediction with ANFIS. We could not obtain satisfactory results and it is shown in figure 14. 18 samples are not enough for accurate prediction with ANFIS. We could not obtain satisfactory results using ANFIS, as shown in figure 14. 

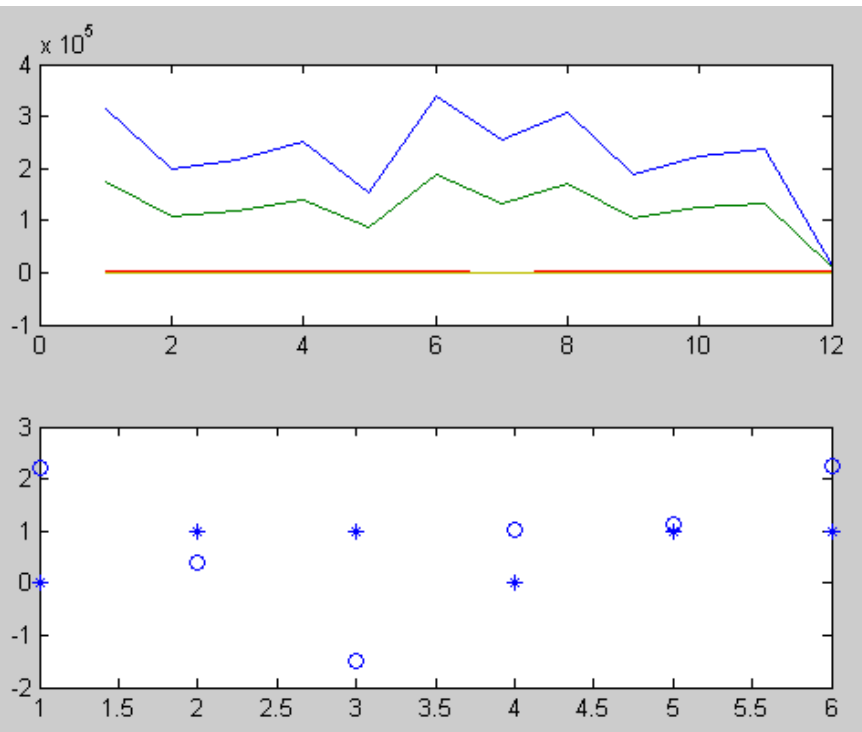

Figure 14. Prediction after training, "o" is the actual value and "**" is the predicted value.

We applied the same real data group for SVM with Linear kernel function. For linear kernel C=1, Epsilon $=\mathrm{e}^{-12}$ and tolerance parameter $=0.100$ are chosen. For Genetic Algorithm; population size is 50 , crossover rate is 0.9 and mutation rate is 0.07 .

Classification of OA and OP using Genetic Algorithm and SVM Algorithm are simulated for various training-test ratios (\%) of all data set such as 50-50 and 66.6-33.3. In order to obtain better network generalization, 4-fold cross validations are also used. Table 2 lists confusion matrix and obtained classification success rate.

Table 2. Confusion Matrix and results of simulated methods.

\begin{tabular}{|c|c|c|c|c|c|c|c|c|c|}
\hline Methods & \multicolumn{3}{|c|}{$\begin{array}{c}\text { Evaluate on } \\
9 \text { training and } 9 \text { testing data }\end{array}$} & \multicolumn{3}{|c|}{ 4-fold cross-validation } & \multicolumn{3}{|c|}{$\begin{array}{c}\text { Evaluate on } \\
12 \text { training and } 6 \text { testing data }\end{array}$} \\
\hline \multirow{3}{*}{ SVM } & $\mathrm{OA}$ & OP & Success Rate $=100 \%$ & OA & OP & $\begin{array}{c}\text { Success } \\
\text { Rate }=88.88 \%\end{array}$ & $\mathrm{OA}$ & OP & $\begin{array}{c}\text { Success } \\
\text { Rate }=83.33 \%\end{array}$ \\
\hline & 4 & 0 & $\mathrm{OA}$ & 8 & 1 & $\mathrm{OA}$ & 3 & 0 & $\mathrm{OA}$ \\
\hline & 0 & 5 & OP & 1 & 8 & OP & 1 & 2 & OP \\
\hline \multirow{3}{*}{ GA } & $\mathrm{OA}$ & OP & Success Rate $=100 \%$ & OA & OP & $\begin{array}{c}\text { Success } \\
\text { Rate }=94.44 \%\end{array}$ & $\mathrm{OA}$ & OP & $\begin{array}{c}\text { Success } \\
\text { Rate }=100 \%\end{array}$ \\
\hline & 4 & 0 & $\mathrm{OA}$ & 9 & 0 & $\mathrm{OA}$ & 3 & 0 & $\mathrm{OA}$ \\
\hline & 0 & 5 & OP & 1 & 8 & OP & 0 & 3 & OP \\
\hline
\end{tabular}




\section{Discussion}

This paper presents an original association of image processing and artificial intelligence methods for a better classification of two studied populations composed of 9 osteoarthritis and 9 osteoporosis samples denoted OA and OP respectively.

Concerning image processing, we have presented a precise assessment technique for the 3D characterization of a complex porous medium such as trabecular bone. Hybrid Skeleton Graph Analysis (HSGA) is a technique which generates structural models of the object. It consists in an efficient combination of curve and surface thinning techniques and considers the local shape of each element that composes the structure of the medium. HSGA can classify each element of the structure as rod-like or plate-like without ignoring the intersections and the termini. Features extracted from the HSGA contain significant topological and morphological information. The HSGA is used to extract mechanical features thanks to a finite element analysis. Twenty-seven features are processed on each of the 18 bone samples. A comparative discriminate analysis using artificial intelligence is applied to identify each of the samples as OA or OP.

For the first test, Adaptive Neuro-Fuzzy Inference System (ANFIS) was used but its success in identifying each of the samples was poor due to lack of samples and many nodes (Figure 14). We then applied Support Vector Machines (SVM) with Linear Polynomial Kernel and obtained a high degree of success. Finally, we used Genetic Algorithm (GA) and obtained correct predictions and $100 \%$ success (Table 2).

The results presented in this paper are consistent with our expectations, since geometrical improvement in the models leads to better and more precise characterization of the structure. The long-term aim of our work is to develop a biomechanical simulation protocol that could be used to virtually characterize the stiffness of trabecular bone samples [16]. This will contribute significantly to detecting bone fragilities simply by acquiring 3D images, for example with a high-resolution in-vivo $\mathrm{CT}$ and simulating mechanical compression using fast and precise $\mathrm{FE}$ models. Associating different techniques with artificial intelligence could be used by physicians to complete their diagnosis efficiently. The drawback of this study is the small number of samples in the populations. Further work is to be carried out on larger sets of trabecular bone samples. 


\section{References}

1. Thiel, E., and Montanvert, A., Shape splitting from medial lines using the 3-4 chamfer distance, In C. Arcelli et al., editors, Visual Form Analysis and Recognition, 537-546.

Plenum, New York, 1992.

2. Gonzalez, R.C., and Woods, R.E., Digital Image Processing. Addison Wesley publisher 50803, 1992.

3. Cruz-Orive, L. M., Estimation of sheet thickness distribution from linear and plate Sections. Biomed, No; 21, pp. 717-730, 1979.

4. Levitz, P., and Tchoubar, D., Disordered porous solids: from chord distributions to small angle scattering. J. Phys, No: II 2, pp. 771-790, 1992.

5. Odgaard, A., and Gundersen, H. J. G., Quantification of connectivity in cancellous bone, with special emphasis on 3D reconstructions. Bone, No : 14, pp. 173-182, 1993.

6. Vogel, H. J., Digital unbiased estimation of the Euler-Poincaré characteristic in different Dimensions. Acta Stereol., No: 16, pp. 97-104, 1997.

7. Hildebrand, T., and Rüegsegger, P., A new method for the model-independent assessment of thickness in three-dimensional images. J. Microsc., Vol. 185, pp 67-75, 1997.

8. Jennane, R., Ohley, W. J., Majumdar, S., and Lemineur, G., Fractal Analysis of Bone X-Ray Tomographic Microscopy Projections, IEEE Tans. on Med. Imag., Vol. 20, No: 5, pp. 443- 449, 2001.

9. Pothuaud, L., Orion, P., Lespessailles, E., Benhamou, C. L., and Levitz, P., A new method for three-dimensional skeleton graph analys is of porous media : application to trabecular bone microarchitecture", Journal of microscopy, Vol. 199 Pt. 2, pp. 149-161, 2000.

10. Aufort, G., Jennane, R., Harba, R., and Benhamou, C. L., A New 3D Shape-Dependent Skeletonization Method. Application to Porous Media. EUSIPCO-2006, Florence, Italy, September 2006.

11. Aufort, G., Jennane, R., and Harba, R., Hybrid skeleton graph analys is of disordered porous media. Application to trabecular bone. In Proc. IEEE Int. Conf. Acou. Spee. Sig. Proc. 2007, pp. II 781-784, Toulouse, France. May 2006.

12. Morgenthaler, D. G., Three-dimensional simple points: serial erosion, parallel thinning and skeletonization. Tech. Rep. TR-1005, 1981. 
13. Pothuaud, L., Rietbergen, B. V., Charlot, C., Ozhinsky, E., and Majumdar, S., A new computational efficient approach for trabecular bone analysis using beam models generated with skeletonized graph technique. Computer Methods in Biomech. Biomed. Eng., vol. 7, no. 4, pp. 205-213, 2004

14. Lenthe, H. G., Stauber, M., and Müller, R., Specimen specific beam models for fast and accurate prediction of human trabecular bone mechanical properties. Bone, 39(6):11821189, 2006.

15. Aufort, G., Jennane, R., Harba, R., Gasser, A., Soulat, D., and Benhamou, C. L., Nouvelle approche de modélisation de milieux poreux. Application à l'os trabéculaire.

GRETSI’05, pp. 429-432, Louvain-la-Neuve, Belgium, September 2005.

16. Aufort, G., Jennane, R., Harba, R., Gasser, A., Soulat, D., and Benhamou, C. L., Mechanical assessment of porous media using hybrid skeleton graph analysis and finite elements. Application to trabecular bone. Proc. EUSIPCO-2007, Poznan, Poland, September 2007.

17. Cherkassky, V., Fuzzy Inference Systems: A Critical Review, Computational Intelligence, Soft Computing and Fuzzy-Neuro Integration with Applications of NATO ASI Series. Computer and Systems Sciences, Springer-Verlag, Chapter 3.2, pp. 177-197. Vol. 162 Berlin, 1998.

18. Jang, J., Self-learning fuzzy controllers based on temporal backpropagation. IEEE Trans. Neural Network, vol.3 No.5, 1992.

19. Vapnik, V., The nature of statistical learning theory. New York: Springer-Verlag, 1995.

20. Siedlecki, W., and Sklansky, J., A Note on Genetic Algorithms for Large-Scale Feature Selection. Pattern Recognition Letters, vol. 10, no. 335-347, Nov. 1989.

21. Sugeno, M., and Kang, G. T., Structure identification of fuzzy model. Fuzzy Sets and Systems 28: 15-33, 1988.

22. Sun, C.T., Rulebase structure identification in an adaptive-network-based fuzzy inference system. IEEE Trans. Fuzzy Systems, vol.2, pp. 64-73, 1994.

23. Takagi, T., and Sugeno, M., Derivation of fuzzy control rules from human operator's control actions. Proceeding s of the IFAC Symposium on Fuzzy Information, Knowledge Representation and Decision Analysis, pp. 55-60, 1983.

24. Übeyl, E. D., and Güler, I., Automatic detection of erythemato-squamous diseases using adaptive neuro-fuzzy inference systems. Computers in Biology and Medicine 35 (5): 421433, 2005. 
25. Takagi, T., and Sugeno, M., Fuzzy identification of systems and its applications to modeling and control. IEEE Transactions on Systems, Man and Cybernetics, vol. SMC15, pp.116-132, 1985.

26. Fogelman, S. F., and Herault, J., Neuro-computing: Algorithms, Architectures and Applications. NATO ASI Series in Systems and Computer Science, Springer, 227-236. New York, 1990.

27. Broomhead, D.S. And Lowe, D., Multivariable functional interpolation and adaptive networks. Complex Syst. 2, pp. 321-355, 1988.

28. Aronszajn, N., Theory of reproducing kernels. Trans. Amer. Math. Soc. 686:337-404, 1950.

29. Girosi, F., An equivalence between sparse approximation and Support Vector Machines. A.I. Memo 1606, MIT Artificial Intelligence Laboratory, 1997.

30. Wahba, G., Spline Models for Observational Data. Series in Applied Mathematics. Vol. 59, SIAM, Philadelphia, 1990.

31 Kilic, N. Ucan, O.N., and Osman, O., Colonic Polyp Detection in CT Colonography with Fuzzy Rule based Template Maching. JOMS, Vol. 33:9-18, 2009.

32. Peña-Reyes, C. A., and M. Sipper, Evolutionary Computation in Medicine: an Overview. Artificial Intelligence in Medicine, 2000. 19:1-23.

33. Koza, J. R., Keane, M.A., and Streeter, M., Evolving Inventions. Scientific American, February 2003:52-59.

34. Miller, M.T., Jerebko, A. K., Malley, J. D., and Summers, R. M., Feature Selection for Computer-Aided Polyp Detection using Genetic Algorithm. SPIE Medical Imaging. 2003.

35. Pei, M., Ding, Y., Punch, W.F., and Goodman, E.D., Genetic Algorithms For Classification and Feature Extraction, Classification Society Conference , June 1995.

36. Aufort, G., Jennane, R., Harba, R., and Benhamou, C. L., Shape classification techniques for discrete 3D porous media. Application to trabecular bone. IEEE-EMBC 2007, August 2007.

37. Ulrich D., Rietbergen, B. V., Weinans, H., and Rüegsegger, P., Finite element analysis of trabecular bone structure: a comparison of image-based meshing techniques. J. biomech., 31(12):1187-1192, 1998.

38. Bayraktar, H. H., Nonlinear micro finite element analysis of human trabecular bone. Circle 141 - Abaqus Inc., pp. 22-25, 2004. 
39. Reinders, F.,. Jacobson, M. E. D., and Post, F. H., Skeleton graph generation for feature shape description. In Proc. Data Visualization, pp. 73-82, 2000.

40. Lorensen, W. E., and Cline H. E., Marching cubes: a high resolution 3D surface construction algorithm. Computer Graphics, 21:163-169, 1987.

41. T. Hilderbrand and P. Ruegsegger, A new method for the model independent assessment of thickness in threedimensional images. Jour. of Microscopy, 185:65-67, 1997

42. Bourne, B. C., Marjolein, C.H., and Meulen, V.D., Finite element models predict cancellous apparent modulus when tissue modulus is scaled from specimen ct-attenuation. J Biomech., 37(5):613-621, May 2004.

43. Hoshen, J., and Kopelman, R., Percolation and cluster distribution i. cluster multiple labeling technique and critical concentration algorithm. Phys. Rev. B, 14:3438-3445, October 1976. 